associated with CV events increased by $73 \%$ (40.7\% v $70.6 \%)$, largely due to a $600 \%(0.3 \%$ v $2.1 \%)$ increase in acute myocardial infarction. Lastly, negative clinical outcomes associated with renal toxicity increased by $433 \%$ (1.5\% v $8.0 \%)$, with a $740 \%(0.5 \% \vee 4.2 \%)$ increase in acute renal failure being the most substantial.

Conclusion: These findings suggest that prescribing of NSAIDs among OA patients is associated with an increase in negative clinical outcomes. This suggests that new treatment options other than NSAIDs should be evaluated.

References:

[1] van Laar M, et al. Pain treatment in arthritis-related pain: beyond NSAIDs. Open Rheumatol J. 2012;6:320-330.

[2] Nissen SE, et al. Cardiovascular safety of celecoxib, naproxen, or ibuprofen for arthritis. NEJM 2016;2519-2529.

Disclosure of Interests: Stuart Silverman Consultant of: Pfizer and Eli Lilly for this project., Speakers bureau: Amgen, Radius, James Rice Consultant of: Pfizer and Eli Lilly have funded this project., Alan White Consultant of: Pfizer and Eli Lilly have funded this project., Nguyen Le Consultant of: Pfizer and Eli Lilly have funded this project., Michael Somma Consultant of: Eli Lilly and Pfizer have funded this project., Craig Beck Shareholder of: Pfizer, Employee of: Pfizer, Rebecca Robinson Shareholder of: Eli Lilly, Employee of: Eli Lilly, Patricia Schepman Shareholder of: Pfizer, Employee of: Pfizer

DOI: 10.1136/annrheumdis-2020-eular.3716

\section{FRI0424 PROGRESSION OF PAIN, STIFFNESS, FUNCTION CHANGES AND ULTRASOUND DETECTED CHANGES IN THE GROUP OF 151 PATIENTS WITH HAND OSTEOARTHRITIS OVER THREE YEARS}

O. Sleglova ${ }^{1}$, O. Růžičková ${ }^{1}$, K. Pavelka ${ }^{1}$, L. Šenolt ${ }^{2} .{ }^{1}$ Institute of Rheumatology Prague, Department of Rheumatology, 1st Medical Faculty, Charles University Prague,, Prague, Czech Republic; ${ }^{2}$ Institute of Rheumatology, Department of Rheumatology, 1st Medical Faculty, Charles University Prague,, Prague, Czech Republic

Background: Hand osteoarthritis ( $\mathrm{HOA})$ is a common and frequent cause of pain. HOA is a heterogeneous group of disorders with two main subsets including non-erosive and erosive disease. Few studies demonstrated inflammatory ultrasound changes and more severe clinical symptoms in patients with erosive compared with non-erosive disease, however the results are inconsistent.

Objectives: The aim of this study was to evaluate progression of pain, stiffness, physical impairment and ultrasound features in patients with erosive and non-erosive HOA in a three years longitudinal study.

Methods: Consecutive patients with symptomatic HOA fulfilling the American College of Rheumatology (ACR) criteria were included in this study. Joint pain and swelling were assessed. Patients reported joint pain on $100 \mathrm{~mm}$ visual analogue scale (VAS). Pain, joint stiffness and disability were assessed by the Australian/Canadian OA hand index (AUSCAN). Erosive disease was defined by at least one erosive interphalangeal joint. Synovial hypertrophy and power Doppler signal (PDS) were scored with ultrasound. Synovitis was graded on a scale of 0-3 and osteophytes were defined as cortical protrusions seen in two planes. Patients were examined at baseline and at the first, second and third year of follow-up.

Results: Altogether, 151 patients (16 male) with symptomatic nodal HOA were included in this study and followed between April 2012 and January 2020. Out of these patients, 84 ( 4 male) had erosive disease. The disease duration $(p<0.05)$ was higher in patients with erosive compared with non-erosive disease.

Pain reported on VAS was significantly higher $(p<0.01)$ in patients with erosive compared with non-erosive disease at baseline. Progression of pain after the third year of follow up was significantly higher in patients with erosive disease $(p<0.01)$. The number of painful and clinically swollen joints $(p<0.05)$ was significantly higher in patients with erosive compared with non-erosive disease at baseline. It fluctuated over the second and third year of follow up, but it still remained statistically higher $(p<0.01)$ at the third year of follow up in patients with erosive disease.

According to the AUSCAN, patients with erosive compared with non-erosive disease had more pain $(p<0.01)$ and stiffness $(p<0.01)$ at baseline. Pain $(p<0.05)$, stiffness and also function $(p<0.05)$ worsened in patients with erosive compared with non-erosive disease at the third year of follow up.

US-detected pathologies such as gray-scale synovitis $(p<0.001)$, intensity of PDS $(p<0.01)$ and number of osteophytes $(p<0.01)$ were significantly higher in patients with erosive compared with non-erosive disease at baseline. There were improvements in gray-scale synovitis total score and intensity of PDS in patients with non-erosive disease while patients with erosive disease worsened after the second and third year of follow up. US-detected gray-scale synovitis $(p<0.001)$, intensity of PDS $(p<0.01)$ remained significantly higher in patients with erosive compared with non-erosive disease after the third year of follow up. On the other hand, the progression of US-determined osteophyte formation was observed in both groups but was significantly higher $(p<0.05)$ in patients with erosive compared with non-erosive disease after the third year of follow up.

Conclusion: The findings of this study show that pain and number of painful and clinically swollen joints associated with US-detected synovial changes and osteophyte formation is more severe in patients with erosive HOA than in patients with non-erosive disease. In addition, osteophyte formation is more likely to progress independent of synovial inflammation.

\section{References:}

[1] Meersseman P, Van de Vyver C, Verbruggen G, et al. Clinical and radiological factors associated with erosive radiographic progression in hand osteoarthritis. Osteoarthritis and Cartilage 2015;23:2129-2133.

Acknowledgments: This work was supported by the project MHCR 023728 and AZV No. 18-00542.

Disclosure of Interests: Olga Sleglova: None declared, Olga Růžičková: None declared, Karel Pavelka Consultant of: Abbvie, MSD, BMS, Egis, Roche, UCB, Medac, Pfizer, Biogen, Speakers bureau: Abbvie, MSD, BMS, Egis, Roche, UCB, Medac, Pfizer, Biogen, Ladislav Šenolt: None declared

DOI: 10.1136/annrheumdis-2020-eular.2055

\section{FRI0425 \\ EVALUATION OF THE DOYLE INDEX AS A MEASURE OF PAIN SENSITIZATION IN PERSONS WITH HAND OSTEOARTHRITIS: EXPLORATORY ANALYSES FROM THE NOR-HAND STUDY}

P. Steen Pettersen ${ }^{1,2}$, T. Neogi $^{3}$, K. Magnusson ${ }^{1}$, B. Slatkowsky-Christensen ${ }^{1}, \mathrm{H}$. B. Hammer ${ }^{1,2}$, T. Uhlig ${ }^{1,2}$, T. K. Kvien ${ }^{1,2}$, I. K. Haugen ${ }^{1}{ }^{1}$ Diakonhjemmet Hospital, Rheumatology, Oslo, Norway; ${ }^{2}$ University of Oslo, Faculty of Medicine, Oslo, Norway; ${ }^{3}$ Boston University School of Medicine, Rheumatology, Boston, United States of America

Background: Pressure pain threshold (PPT) is a measure of pain sensitization; altered pain mechanisms in the peripheral and/or central nervous system causing increased pain sensitivity. PPT testing may be a useful tool to classify pain phenotypes but requires special equipment not available in the clinic. The Doyle index (DI) is a clinical measure of joint tenderness upon palpation. It is considered as an outcome measure of pain and disease activity in hand OA and is a potential alternative to PPT. It is unclear if joint tenderness is related to pain sensitization, as joint tenderness could reflect pure nociceptive pain without sensitization.

Objectives: Using data from the Nor-Hand study we will explore how DI performs as a measure of pain sensitization in hand OA by examining associations and agreements between DI and PPT at joint level, and correlations between PPT values and DI sum score at person level.

Methods: PPT was tested with a hand-held algometer (FPIX25, $1 \mathrm{~cm}^{2}$ flat rubber tip) at the dorsal side of the most painful DIP/PIP and a non-painful DIP/PIP joint (local PPTs) and left radioulnar joint and mid-portions of the trapezius and tibialis anterior muscle (remote PPTs). Low local PPTs indicate peripheral and/or central sensitization, while low remote PPTs indicate central sensitization. According to DI, tenderness in the bilateral thumb base and finger joints were graded by a rheumatologist by pressing on the lateral joint margins: $0=$ no pain, $1=$ patient complains of pain, $2=$ patient complains of pain and winces, $3=$ patient complains of pain, winces and withdraws joint. We examined whether increasing DI was associated with local PPT using mixed models. To assess agreement between DI and PPT, we categorized PPT of the painful finger joints into a semi-quantitative scale with the same number of categories $(n=4)$ as DI. We identified the cut-offs for the PPT categories that maximized the agreement (weighted kappa) with DI. Finally, we examined Spearman's correlations between DI sum score [range 0-90] and PPTs of local and remote sites.

Results: The majority of the 285 eligible participants were women (88\%) and mean (SD) age was 61 (6) years. Joints with high DI had lower PPT values (Figure 1). We found a linear association of lower PPT with increasing DI for all joints combined (beta $-0.7,95 \% \mathrm{Cl}-0.8,-0.6)$. Similar results were found for the painful joints (beta $-0.8,95 \% \mathrm{Cl}-1.0,-0.6$ ), but weaker for non-painful joints (beta $-0.5,95 \% \mathrm{Cl}-1.0,0.0$ ) where few joints had DI grade 2-3 (Figure 1). The analyses on maximized agreement between DI and the PPT categories gave a weighted kappa equal to 0.32 (Table).

Median (IQR) DI sum score was $9(5,15)$. We found weak inverse correlations between DI sum score and PPT at local (painful finger: $\rho-0.24(95 \% \mathrm{Cl}-0.32$, $-0.16)$, non-painful finger: $\rho-0.22(95 \% \mathrm{Cl}-0.29,-0.11)$ and remote sites (radioulnar joint: $\rho-0.17(95 \% \mathrm{Cl}-0.29,-0.06)$, trapezius: $\rho-0.25(95 \% \mathrm{Cl}-0.36,-0.14)$, tibialis anterior: $\rho-0.20(95 \% \mathrm{Cl}-0.31,-0.09))$.

Conclusion: The DI was associated with lower PPT at painful finger joints. Large variance of PPT within each DI grade resulted in fair agreement. DI of non-painful 